“ (C) 2016 IEEE. Personal use of this material is permitted. Permission from IEEE must be obtained for all other uses, in any current or future media, including

reprinting/republishing this material for advertising or promotional purposes, creating new collective works, for resale or redistribution to servers or lists, or reuse of any copyrighted component of this work in other works." 


\title{
Integration and Operation of an Electrically Small Magnetic EZ Antenna with a High Power Standing Wave Oscillator Source
}

\author{
Eric S. Ramon, Student Member, IEEE, J. Scott Tyo, Fellow, IEEE, Richard W. Ziolkowski, Fellow, IEEE, \\ Michael C. Skipper, Member, IEEE, Michael D. Abdalla, Member, IEEE, Joshua Martin, Member, IEEE
}

\begin{abstract}
The efficacy of the three-dimensional, rectangular magnetic EZ antenna for use with mesoband high power microwave (HPM) sources has been demonstrated previously. It overcomes the typical bulky and massive impedance matching components found currently in most HPM systems, making it an attractive option when space is very limited. However, its extremely compact nature presents practical challenges when dealing with extremely high power sources due to the associated local field enhancements near the feed and the near field resonant parasitic element. This letter presents a fully integrated, highvoltage source and radiating system that has several improvements in the antenna, source, and power system that have not before been demonstrated. The full system includes a ferroelectric generator, standing wave oscillator source, and electrically small antenna $(\mathrm{ka}=0.37)$ operating at $510 \mathrm{MHz}$ that can be packaged inside a $15-\mathrm{cm}$ diameter tube. This small diameter results in a quarter-wavelength-diameter ground plane, and the effects of this small ground plane on the radiation characteristics are explored. The development of a pressurized radome allows for operation at $73.6 \mathrm{kV}$, significantly higher than previous studies. The results in this letter demonstrate that several disparate parts can be brought together to make a self-contained, compact, HPM system.
\end{abstract}

the performance characteristics obtained through numerical simulations.

\section{INTRODUCTION}

High power electromagnetic (HPEM) systems have many uses that range from high power radar systems and intentional electromagnetic interference (IEMI) [1]. In these application it is desired to deliver peak powers up to and in excess of $100 \mathrm{~s}$ of MW. Such systems are typically categorized based on their percent bandwidth (pbw): narrowband ( $11 \% \mathrm{pbw})$, mesoband or moderateband (1\% - 100\% pbw), sub-hyperband (100\% $163.4 \%$ pbw) and hyperband (163.4\% - 200\% pbw) [2], [3]. Where the pbw definition is based on the 2-norm as suggested by Baum and Nitsch [3], [4].

In mesoband HPM applications there are two common methods for creating radiating systems: 1) excite a selfresonant antenna using a wideband transient pulse generator [5], or 2) use a self-contained resonator as the source, which is coupled to an antenna used to radiate the stored energy [6]. In our experience we have found that the former method

E. S. Ramonand R. W. Ziolkowski are with the College of Optical Sciences and the ECE Department at the University of Arizona in Tucson. J. S. Tyo is with the School of Engineering and IT at the University of New South Wales Canberra, Australia. M. C. Skipper and M. D. Abdalla are with ASR Corporation in Albuquerque, NM. J.M. Martin is with the US Army Space and Missile Defense Command in Huntsville, AL. is better suited for applications under $150 \mathrm{MHz}$ when space is extremely valuable, while the latter is suited for higher frequency applications because of the ability to store more energy and achieve higher gain for a given volume. Using the second method in our application we have previously presented work discussing a quarter-wave switched wave oscillator (SWO) source, sometimes referred to as a MATRIX source [2], [7], [8].

The SWO source is a HPM mesoband source with a bandwidth of approximately $10 \%$ that produces a damped sinusoidal output [6]. A basic layout for this source is depicted in Fig. $1 \mathrm{~b}$ The inner and outer conductor form a low impedance transmission line that is terminated by a high impedance load, which in our application is the antenna [9], [10]. The antenna input impedance is designed to be near 100 Ohms in order to achieve a $Q$ of about 20 , which is desirable for these applications [7].

Traditional HPM systems are large and bulky, which causes difficulties in applications that have small maximum requirements on the form factor or weight of the system [1]. For a given source, the best way to reduce the size of the system is to utilize an electrically small antenna. Unfortunately electrically small antennas typically suffer from low efficiencies and are poorly matched to a real input impedance without the assistance of external matching networks [11], [12]. Here we will use the definition for an electrically small antenna $k a<0.5$, where $k=2 \pi / \lambda_{\text {res }}$ [13], [14].

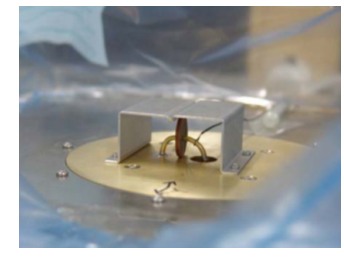

(a)

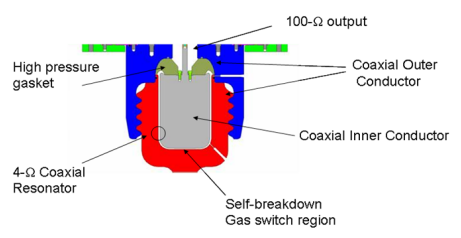

(b)
Fig. 1: (a) A previously built HPM EZ antenna and (b) a cross sectional diagram of a SWO source

One method explored to create efficient ESAs is to fill the space surrounding the antenna with a metamaterial (MTM) shell that is conjugate matched to the antenna. This causes the reactive component of the impedance to approach zero [11], [15]. One specific design of MTM-inspired antennas is the "EZ antenna", which utilizes a capacitively loaded loop (CLL) 
placed in the near-field of a magnetic dipole antenna. These antennas have been shown to have efficiencies of greater than $90 \%$ while maintaining $k a<0.5$ [12], [16], and have been adapted to a SWO source in our earlier work [7], [8]. Here we further shrink the size of the CLL by using a spherical shape instead of an extruded rectangle, we demonstrate the performance of the EZ antenna over a very small ground plane $(\lambda / 4$-diameter $)$, and we integrate the antenna with a pressurized radome to increase the maximum voltage above the $10 \mathrm{kV}$ that we demonstrated previously.

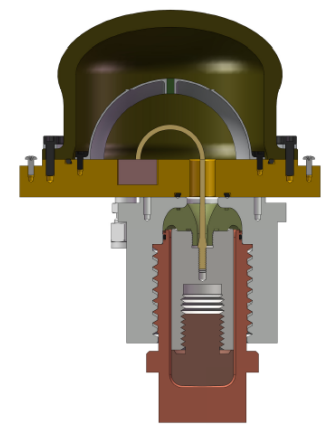

Fig. 2: A cross section of a 3D rendering of the HPM system with a SWO source, spherical EZ antenna, and radome

This paper presents a modified EZ antenna geometry with a spherical near-field element. A cross section of this antenna, with the source and radome, is shown in Fig. 2. The radome contains $S F_{6}$ gas at pressures up to 50 psi to insulate the feed-point of the EZ antenna. The radome is fabricated from ULTEM 2300 and is mounted on the ground plane. Some of the dimensions of the radome and the EZ antenna are listed in Table I The thickness of the radome was chosen such that it maintained a factor of safety of 5 at 50 psi.

\begin{tabular}{lc} 
Dimension & Value $[\mathrm{cm}]$ \\
\hline Overall Height & 7.56 \\
Thickness (Spherical Section) & 0.86 \\
Thickness (Cylindrical Section) & 0.62 \\
Inner Height & 6.70 \\
EZ Antenna & 4.04 \\
Height &
\end{tabular}

TABLE I: Table of mechanical parameters of the EZ antenna

The height of the radome was chosen to maintain $2.54 \mathrm{~cm}$ of clearance between the top of the EZ antenna and the dome. This distance was arbitrarily chosen to minimize the interaction between the radome and the EZ antenna. It is likely that the height of the radome can be reduced while maintaining the electrical performance of the antenna. A future design might produce a radome that is only slightly larger than the EZ antenna, and its shaping and/or thickness could be tailored specifically to optimize the radiation pattern.

\section{EXPERIMENTAL RESULTS}

Two experimental tests were done using the spherical EZ antenna. The first experiment was a high power test where the SWO was charged to $73.6 \mathrm{kV}$ with the EZ antenna mounted over a 24"x24" ground plane, as shown in Fig. 3a. The electric field amplitude and spectrum were measured $1 \mathrm{~m}$ from the ground plane in the broadside direction for this experiment. The second experiment was mapping the electric field pattern of the antenna. In this experiment the large ground plane was removed and replaced with a circular ground plane with a radius of approximately $15 \mathrm{~cm}$ (approximately the same size as the base of the EZ antenna). An extruded tube was added that extended $1 \mathrm{~m}$ below the ground plane to cover the SWO. The EZ antenna in this configuration is shown in Fig. 3b. The electric field was mapped by measuring it every $22.5^{\circ}$ in the E- and H-plane at a distance of $1.5 \mathrm{~m}$ from the antenna.

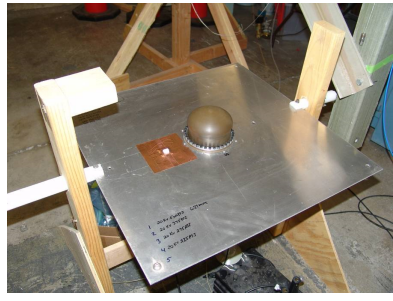

(a)

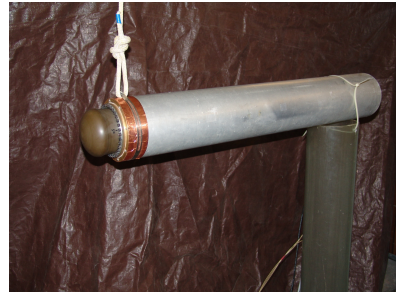

(b)
Fig. 3: The spherical EZ antenna in a radome with (a) a 24 "x24" ground plane and (b) small ground plane and tube backing

\section{A. High Power Electric Field Measurement}

To make the measurements for the high power test, the antenna system was placed in an anechoic chamber. A Prodyne Technologies AD-70 D-dot sensor was used to measure the radiated electric field, and the signal from the sensor was recorded using an oscilloscope. To measure the voltage signal applied to charge the SWO, a Northstar high voltage sensor was used. The radome surrounding the EZ antenna was pressurized to 35 psi with SF6 gas to raise the dielectric strength of the volume surrounding the input of the antenna. To excite the SWO with charge voltages beyond $70 \mathrm{kV}$, a ferroelectric generator (FEG) was used. The FEG is a pulsed power source capable of delivering large voltages in a short time and consists of a piezoelectric ceramic to which a large electric field is applied to create a large charge in the material. Then an explosive charge is used to shock the piezoelectric material and release the stored charge [17]. The cavity of the SWO was pressurized to $2000 \mathrm{psi}$ with hydrogen gas in order to raise the breakdown voltage of the switch. The voltage signal applied to the SWO is shown in Fig. 4. The excitation signal is a nearly linear, relatively slowly charging pulse followed by an extremely rapid discharge when the hold-off voltage of the switch is reached. In this experiment the charge voltage of the source reached $73.6 \mathrm{kV}$.

The measured electric field in the time and frequency domain for this high power test is shown in Fig. 5 The peak electric field amplitude measured $1 \mathrm{~m}$ from the ground plane was $10.6 \mathrm{kV} / \mathrm{m}$. This is over a magnitude larger than our previous reported field strengths from a SWO EZ antenna HPM system [7] $(720 \mathrm{~V} / \mathrm{m}$ at $10 \mathrm{kV}$ charge voltage). The components of the FEG and source-antenna system are shown in Fig. 6. The entire system is extremely compact; a fully 


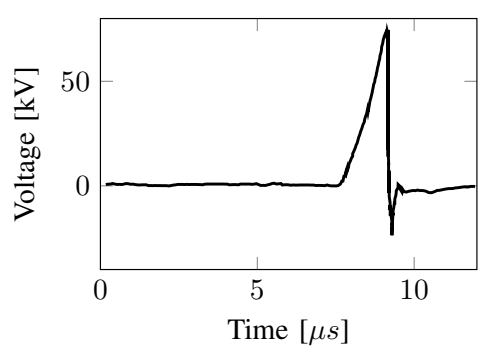

Fig. 4: The charge voltage signal of the source

packaged system would occupy a spherical volume with radius less than $12 \mathrm{~cm}$.

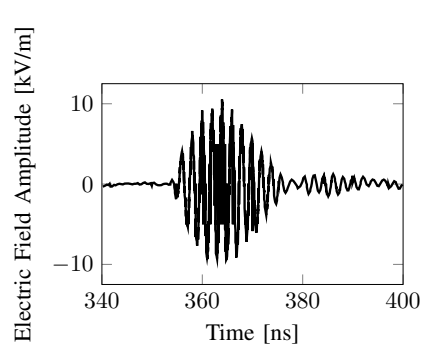

(a)

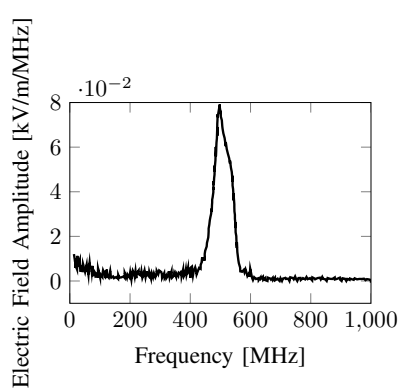

(b)
Fig. 5: The measured electric field $1 \mathrm{~m}$ from the ground planein the (a) time and (b) frequency domain when the SWO was charged to $73.6 \mathrm{kV}$

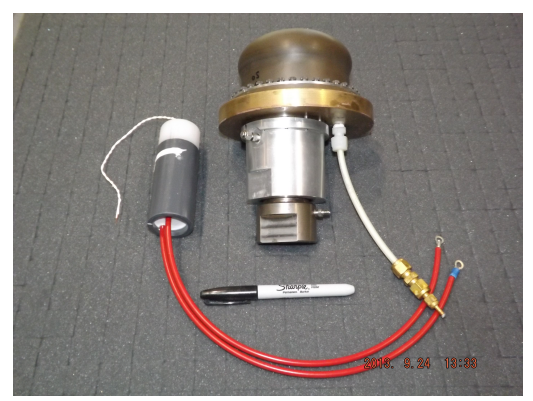

Fig. 6: SWO source, EZ antenna, and FEG shown with a size reference (standard size sharpie pen)

\section{B. Field Mapping Measurements}

The electric field pattern of the system was mapped using the the configuration shown in Fig. $3 \mathrm{~b}$ The EZ antenna was placed over a cylindrical tube that could completely contain the SWO and FEG. Because of the explosive nature of the FEG, this test was performed using a function generator to drive the antenna directly. It is apparent that the ground plane in this experiment is approximately the size of the spherical EZ antenna itself, which is much smaller than what was used in the high power experiments. The measurements were made every $22.5^{\circ}$ in the E- and $\mathrm{H}$-plane of the antenna at a distance of $1.5 \mathrm{~m}$. Fig. $3 \mathrm{~b}$ shows the system oriented horizontally with a dielectric rope suspending the antenna end of the system and a pivot point at the back end of the tube. This setup was used so the system could be rotated about the EZ antenna to map the electric field magnitude at the different field angles.

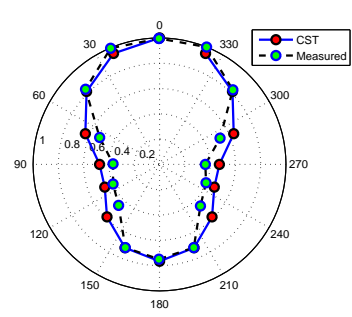

(a)

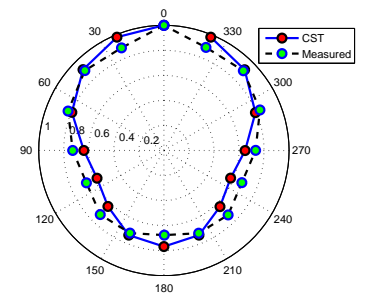

(b)
Fig. 7: Measured (black dashed) and simulated (blue) normalized electric field pattern in the plane (a) perpendicular to the electric field (and containing the axis of the cylinder in Fig. 3b) and (b) parallel to the electric field (and containing the axis of the cylinder in Fig. 3b

The normalized measured electric field pattern in the planes parallel and perpendicular to the electric field are shown in Fig. 7 As we would expect from the symmetry of the system, the peak radiated fields are in the broadside direction of the antenna. The radiation pattern is close to being constant in the plane parallel to the electric field, while in the orthogonal plane the pattern resembles the typical dipole radiation pattern, which is expected given that the magnetic EZ antenna behaves in a similar manner to a magnetic dipole [18].

\section{CST Simulation Results}

We also developed a numerical simulation in CST Microwave Studio [19] to allow us to probe additional behavior of the system that isn't easily measured in the experimental setup. To simulate the system performance, we used a 3D model of the SWO source and the spherical EZ antenna that is similar to the model in Fig 2 with simplifications, such as excluding the radome and using a flat ground plane without any mounting features.

When the model was first simulated, the resonant frequency of the MATRIX source and the EZ antenna were not well matched. In order to correct this, we adjusted the thickness of the G-10 capacitor that fills the gap of the CLL-based near field resonant parasitic element of the antenna to tune its resonance frequency. This is the same method that was used to tune the EZ antenna to the SWO in the built system [7]. Additionally, we adjusted the vertical spacing between the inner and outer conductor of the SWO (where the breakdown switch is located) in order to tune the resonant frequency of the source, which is an adjustment that is used when tuning the built SWO [20]. This tuning is required because the simplifications of the CST models mean they cannot capture the fine detail in the originally built system. Therefore, we would expect them to behave slightly differently than the built elements, which leads to the necessity of tuning the simluations and experimental structures independently.

After tuning the two components of the system in the model, we ran a high power simulation to mimic the experiment from 
section II-A by applying a linearly increasing voltage with peak voltage of $74.6 \mathrm{kV}$. The resulting electric field in the time and frequency domain are shown in Fig. 8

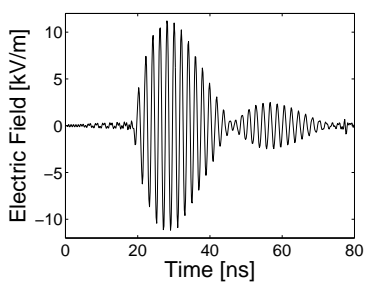

(a)

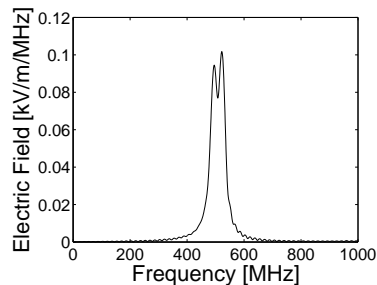

(b)
Fig. 8: Electric field measured $1 \mathrm{~m}$ from the antenna feed as a function of (a) time and (b) frequency

Comparing Fig. 8 to Fig. 5, we can see that the experimental and simulation results agree quite well for the high power experiments measuring the electric field amplitude in the far field. Both time domain signals (Figs. 8a and 5a have peak electric fields just above $10 \mathrm{kV} / \mathrm{m}$, and both evolve similarly over time. The main difference in these signals is that the experimental time domain electric field is damped slightly more than the CST simulations predict. The spectral density plots for the simulation and experiment (Figs. $8 b$ and $5 b$ show that there is about a $20 \%$ difference in the peak spectral density between the two sets of data. Additionally, the CST results show a splitting of the peak resonance in the spectral density plot, which is likely caused by the slower decay of the temporal electric field amplitude.

The two sets of results do not match perfectly, which is to be expected since it is difficult to create models that perfectly simulate some of complex processes in these pulsed power systems due to the complex field and loss behavior near the breakdown region in the SWO when the source is excited. These processes are likely oversimplified in our CST modeling of the switch excitation of the SWO, which will lead to discrepancies between the experimental and simulated results. Regardless of this, we can see that using the CST simulations allows us to get a reasonable prediction of the performance of a system before it is built.

Using the smaller ground plane configuration, we also measured the electric field pattern in the plane parallel and perpendicular to the electric field (and containing the axis of the cylinder shown in Fig. 3b]. The simulated normalized electric field pattern is plotted along with the measured pattern in Fig. 7 It can be seen that there is good agreement between the two sets of data.

\section{CONCLUSION}

In this paper we make the first demonstration of a fully integrated, extremely compact HPM system operating at 510 $\mathrm{MHz}$ that includes the prime power, pulsed power, microwave source, and antenna element, all of which can fit inside a cylindrical tube of diameter less than $15 \mathrm{~cm}(\lambda / 4)$. We showed results from experiments measuring the peak radiated fields, as well as a mapping of the electric field pattern radiated by the antenna. We also compared these results to CST simulations to verify the antenna's behavior. By using a small radome, we were able to fill the space surrounding the antenna with SF6 gas, which effectively raised the dielectric strength near the antenna. This allowed us to raise the charge voltage of the source from around $10 \mathrm{kV}$ that was used in our previous experiments [7] to $73.6 \mathrm{kV}$, resulting in over a factor of 10 increase in peak radiated electric field strength.

\section{REFERENCES}

[1] D. Giri, High-Power Electromagnetic Radiatiors: Nonlethal Weapons and Other Applications. Cambridge, Massachusetts, and London, England: Harvard University Press, 2004.

[2] D. Giri, F. Tesche, and C. E. Baum, "An overview of high-power electromagnetic (HPEM) radiating and conducting systems," Circuit and Electromagnetic System Design Notes, Tech. Rep. 50, Februrary 2006.

[3] D. Giri and F. Tesche, "Classification of intentional electromagnetic environments (IEME)," IEEE Trans. Electromagn. Compat., vol. 46, no. 3, pp. 322-328, August 2004.

[4] C. E. Baum and D. H. Nitsch, "Band ratio and frequency-domain norms," Interaction Note 584, May 2003.

[5] M. M. Armanious, S. V. B. Olaya, J. S. Tyo, M. C. Skipper, M. D. Abdalla, L. L. Altgilbers, and A. Bryan, "An electrically small conical folded dipole antenna for use as a compact, self-resonant mesoband high-power microwave source," IEEE Trans. Antennas Propag., vol. 62, no. 12, December 2014.

[6] W. Prather, C. Baum, R. Torres, F. Sabath, and D. Nitsch, "Survey of worldwide high-power wideband capabilities," IEEE Trans. Electromagn. Compat., vol. 46, no. 3, pp. 335-344, August 2004.

[7] J. Ng, R. W. Ziolkowski, J. S. Tyo, M. C. Skipper, and M. D. Abdalla, "An efficient, electrically small, three-dimensional magnetic EZ antenna for HPM applications," IEEE Trans. Plasma Sci., vol. 40, no. 11, pp. 3037-3045, Nov. 2012.

[8] J. Ng, R. W. Ziolowski, J. S. Tyo, M. C. Skipper, and M. D. Abdalla, "Driven, metamaterial-inspired, 3d magnetic EZ antenna for mesoband HPM applications," in Antennas and Propagation Society International Symposium APSURSI, 2012 IEEE, July 2012, pp. 1-2.

[9] C. E. Baum, "Switched oscillators," Circuit and Electromagnetic System Design Notes, Air Force Research Laboratory, Tech. Rep. 45, 2000.

[10] D. Giri, F. M. Tesche, M. D. Abdalla, M. C. Skipper, and M. Nyffeler, "Switched oscillators and their integration into helical antennas," IEEE Trans. Plasma Sci., vol. 38, no. 6, pp. 1411-1426, June 2010.

[11] R. W. Ziolkowski and A. D. Kipple, "Application of double negative materials to increase the power radiated by electrically small antennas," IEEE Trans. Antennas Propag., vol. 51, no. 10, pp. 2626-2640, October 2003.

[12] R. W. Ziolkowski and A. Erentok, "Metamaterial-based efficient electrically small antennas," IEEE Trans. Antennas Propag., vol. 54, pp. 2113-2130, July 2006

[13] L. Chu, "Physical limitations of omnidirectional antennas," Research Laboratory of Electronics at Massachusetts Institute of Technology, Tech. Rep. 64, May 1948.

[14] H. Wheeler, "Fundamental limitations of small antennas," Proc. of the IRE, vol. 35, pp. 1479-1484, December 1947.

[15] R. W. Ziolkowski, "Applicaitons of metamaterials to realize efficient electrically small antennas," in Proc. IEEE Int. Workshop on Antenna Technology: Small Antennas and Novel Metamaterials, March 2005, pp. 7-9.

[16] R. W. Ziolkowski, C. Lin, J. A. Nielsen, M. H. Tanielian, and C. L. Holloway, "Design and experimental verificaiton of a 3D magnetic EZ antenna at $300 \mathrm{MHz}$," IEEE Antennas Wireless Propag. Lett, vol. 8, pp. 989-993, 2009.

[17] E. Alberta, B. Michaud, W. Hackenberger, B. Freeman, D. Hemmert, A. Stults, and L. Altgilbers, "Development of ferroelectric materials for expolsively driven pulsed-power systems," in Pulsed Power Conf., 2009 IEEE, July 2009, pp. 161-166.

[18] A. Erentok and R. W. Ziolkowski, "Metamatrieral-inspired efficient electrically small antennas," IEEE Trans. Antennas Propag., vol. 56, no. 3, pp. 691-707, March 2008.

[19] (2013) Cst studio suite. [Online]. Available: http://www.cst.com/2013/

[20] M. Armanious, "Design and analysis of a high power moderate band radiator using a switched oscillator," Ph.D. dissertation, University of Arizona, 2010. 